\title{
Wind Versus Storage Allocation for Price Management in Wholesale Electricity Markets
}

\author{
Amin Masoumzadeh, Ehsan Nekouei, Member, IEEE, and Tansu Alpcan, Senior Member, IEEE
}

\begin{abstract}
This paper investigates the impacts of installing regulated wind and electricity storage, by a state-owned (government) entity, on average price and price volatility in electricity markets. A stochastic bi-level optimization model is developed which computes the optimal sizing of new wind and battery capacities by minimizing a weighted sum of the average market price and price volatility. A fixed budget is allocated on wind and battery capacities in the upper level problem. The operation of strategic/regulated generation, storage and transmission players is simulated in the lower level problem using a stochastic (Bayesian) Cournot-based game model. The Australia's National Electricity Market (NEM), which is experiencing occasional price peaks, is considered as the case study. Our simulation results quantitatively illustrate that the regulated wind is more efficient than storage in reducing the average price, while the regulated storage more effectively reduces the price volatility. According to our numerical results, the storage-only solution reduces the average price at most by $\mathbf{9 . 4 \%}$, and the wind-only solution reduces the square root of price volatility at most by $39.3 \%$. However, an optimal mixture of wind and storage can reduce the mean price by $17.6 \%$ and the square root of price volatility by $48.1 \%$. It also increases the consumer surplus by $1.52 \%$. Moreover, the optimal mixture of wind and storage is a profitable solution unlike the storage-only solution.
\end{abstract}

Index Terms-Electricity market, Bi-level optimization model, Average price, Price volatility, Regulated wind-storage firm.

\section{INTRODUCTION}

$\mathbf{H}$ IGH levels of market power is one of the main reasons behind high electricity prices in highly concentrated electricity markets, such as Australias National Electricity Market (NEM) [1], [2]. For example, the electricity prices have increased in average by $200 \%$ in NEM during 2015-2018 mainly due to exercise of market power by strategic power plants. High price volatility and mean price levels have negative impacts on all parties involved in the electricity markets, e.g., generators and consumers. Price volatility imposes large financial risks on the market participants by increasing the future price prediction uncertainty [3]. Extreme levels of price volatility may also lead to market suspension, for example, NEM becomes suspended if the sum of spot prices during a period is above the Cumulative Price Threshold. On the other hand, high levels of mean wholesale electricity prices lead to higher retail prices, i.e., impose high cost on consumers. For example, average household power bills increased almost 16 percent after Hazelwood coal plant closure in Victoria [4].

Recently, limited government intervention has been proposed as a solution which may pave the way towards more efficient markets as the private sector is likely to act slowly due to regulatory,

We also acknowledge this work was supported in part by the ARC Discovery Project DP140100819. A. Masoumzadeh and T. Alpcan are with the Department of Electrical \& Electronic Engineering, University of Melbourne, Australia, and E. Nekouei is with KTH Royal Institute of Technology, Sweden. Emails: amin.masoumzadeh@unimelb.edu.au, tansu.alpcan@unimelb.edu.au, nekouei@kth.se institutional, or other barriers [5]. For example, in 2017, South Australia (SA) government took an initiative to improve the SAs electricity market structure by installing $100 \mathrm{MW}$ (125 MWh) electricity storage devices manufactured by Tesla. Following the success of the SA battery project, Victoria has also secured an agreement to build its own Tesla battery near the town of Stawell [6].

In such cases, the government may choose to intervene and install regulated wind and storage capacities, which have short construction periods, to increase the competition in the market (which leads to increase in consumer surplus) and reduce the market power as well as the electricity prices [7]. Note that average prices and volatility levels after the government intervention still must allow competing firms to make money.

Our goal in this paper is to find the optimal state-owned wind and storage capacity balance which, given a limited budget, minimizes a weighted sum of the mean price and the price volatility. We note that the market price in an electricity market is a stochastic process due to the random nature of renewables and stochastic nature of the electricity demand. Thus, it is desirable to simultaneously control both the first moment of the price (its mean) and its second moment (its volatility). Note that minimizing only the mean price (the volatility) can result in increasing volatility (mean). Therefore, our approach can be interpreted as a risk-sensitive solution which aims at minimizing the weighted sum of the mean price and the price volatility. Although, regulated storage has other positive effects on the electricity networks, e.g., improving the stability, in this paper we only focus on its effect on the electricity market prices. The model is applied to the fivestate NEM market and is calibrated with realistic data from year 2016.

\section{A. Contributions}

This paper studies the optimal allocation of storage and wind for minimizing a linear combination of the average price and the square root of price volatility, which have the same scale of $\$ / \mathrm{MWh}$ in our study. The contributions of this paper are summarized as follows:

- A bi-level optimization model is proposed to optimally allocate a fixed budget between regulated wind and storage capacities to minimize the weighted sum of average price and price volatility, in a multi-region wholesale electricity market.

- In the upper level problem, the weighted sum of average price and price volatility is minimized by allocating a fixed budget on regulated wind and storage capacities in that region.

- In the lower level problem, the non-cooperative market interaction between strategic/regulated generation, storage and transmission players and a regulated wind-storage player is modeled as a stochastic (Bayesian) Cournot-based game. The existence of Bayesian Nash Equilibrium (Bayes-NE) [8] is established 
for the lower level problem, which includes nonlinear inverse demand functions.

- Our bi-level optimization model is converted into an equivalent single level problem, in which the optimal capacities of wind and storage are calculated via a line search algorithm.

- The impacts of wind and storage on average price and price volatility are quantified in an analytical game-theoretic market model. Our numerical results indicate that the regulated wind is more efficient than storage in reducing the average price whereas the regulated storage more effectively reduces the price volatility.

- The lifetime cost-benefit analysis of investment on wind and storage, considering a year with hourly resolution, is conducted in our study. It is observed that when the storage only solution is not economical, the investment on a mixture of wind and storage capacities may make profit for the regulated firm. Besides, the optimal mixture of regulated wind and storage capacities is more efficient in increasing the consumer surplus than the storage-only solution.

\section{B. literature Review}

The problem of storage allocation in presence of intermittent renewable energy generation in electricity networks has been studied in [9]-[14] using cost minimization approaches, and in [15]-[20] using profit maximization objectives.

Facilitating the integration of renewable resources, the potential value of energy storage in power systems with renewable generation is evaluated by minimizing the total operation cost in the network in [9]. The optimal operation and sizing of the storage systems is studied by minimizing the cost of the system in [10]. The storage allocation in renewable integrated power systems is studied in [11] and [12] under deterministic and stochastic wind models, respectively. To accommodate the integration of renewable generation, bi-level optimization models are also proposed to determine the optimal allocation and operation of energy storage systems in [13] and of battery energy storage systems in [14], in which the upper level problem minimizes the storage system cost and the lower level problem implements the power flow in the network. Note that these works are based on cost minimization models and do not investigate the market interplay between storage, renewable generators and other firms.

Assuming the storage firms as price takers in the market, the optimal operation of storage firms in renewable integrated systems is determined by maximizing the profit from energy arbitrage and regulation services in [15], by maximizing the energy arbitrage profit in day-ahead and hour-ahead markets in [16], and by maximizing their energy and reserve profit in day-ahead and hourahead markets in [17]. Assuming the storage firms as price maker players in the market, the optimal charge/discharge operation of the storage devices, and the optimal operation and size of the storage devices are determined in [18] and [19], respectively, treating the price bids of market participants other than the storage players as exogenous inputs. The market operation behavior of all generation and storage firms are considered endogenously in a single-region electricity market in [20] using a Cournot-based electricity market model.

The impact of the optimal storage allocation on price volatility reduction in a multi-region electricity market model is studied in [21]. However, studying the joint effect of wind and storage allocation on market price characteristics is missing in the literature. As we show in this paper, wind firms are potentially more efficient than storage firms in reducing the average price and the results of [21] are not applicable when it is desirable to reduce the average price in the market. Therefore, different from the existing work, we consider the problem of managing the average price and the price volatility by optimal allocation of wind and storage capacities.

The rest of the paper is organized as follows. Section II illustrates the system model and the proposed bi-level optimization problem. The solution approach for finding the market equilibrium is presented in Section III. Section IV provides the simulation results and Section $\mathrm{V}$ presents the concluding remarks.

\section{The Problem And Market Model}

We consider a regional electricity market including $\left\{1, \ldots, N_{I}\right\}$ regions (states). Let $\mathcal{N}_{i}^{\text {ig }}$ be the set of intermittent generation firms located in state $i, \mathcal{N}_{i}^{\text {sg }}$ be the set of synchronous generators, such as coal, gas, and hydro power plants, located in state $i, \mathcal{N}_{i}^{\text {s }}$ be the set of storage firms, such as pump-hydro and battery, located in state $i$, and $\mathcal{N}_{i}^{\text {tr }}$ be the set of neighboring states of the state $i$. Since some parameters such as wind and solar power availabilities, which affect the electricity generation, are stochastic, a scenario-based model including $N_{W}$ different scenarios is developed to model the intermittent power generation in the electricity network. The strategies of intermittent and synchronous generators, storage firms, and transmission players as well as the nodal prices are determined by solving a stochastic (Bayesian) Cournot-based game.

In this paper, we present a bi-level optimization framework for optimally allocating a budget on regulated capacities of wind and storage to minimize the weighted sum of average price and price volatility in a single state taking into account the interdependencies to other states in the market. All the market players, which are allowed to be strategic or regulated, with their decision variables, operating limits, and objective functions are introduced in detail in the lower level problem, Section II-B.

Note that the lower level problem in this paper is similar to the one used in [21] but differs in including a regulated wind and storage firm in the market.

\section{A. Upper-level Problem}

In the upper-level optimization problem, we minimize the weighted sum of average price and its standard deviation over the operation horizon $\left\{1, \ldots, N_{T}\right\}$ and scenario set $\left\{1, \ldots, N_{W}\right\}$ at state $i^{*} \in\left\{1, \ldots, N_{I}\right\}$ by allocating a fixed budget on regulated storage and wind generation technologies. The price volatility is measured by the regional price variance [22], i.e., the variance of market price is considered as a measure of price volatility. Market price variance and mean in state $i^{*}$ under a set of scenarios $\left\{1, \ldots, N_{W}\right\}$, i.e., $\operatorname{Var}\left(\left\{P_{i^{*} t w}\right\}_{w}\right)$ and $\mathrm{E}\left(\left\{P_{i^{*} t w}\right\}_{w}\right)$, are defined as:

$$
\begin{aligned}
& \operatorname{Var}\left(\left\{P_{i^{*} t w}\right\}_{w}\right)=\sum_{w}\left(P_{i^{*} t w}(.)\right)^{2} \Psi_{w}-\left(\sum_{w} P_{i^{*} t w}(.) \Psi_{w}\right)_{(1 \mathrm{a})}^{2} \\
& \mathrm{E}\left(\left\{P_{i^{*} t w}\right\}_{w}\right)=\sum_{w} P_{i^{*} t w}(.) \Psi_{w}
\end{aligned}
$$

where $\Psi_{w}$ is the probability of scenario $w$, and $P_{i^{*} t w}($.$) repre-$ sents the market price in state $i^{*}$ at time $t$ under the scenario $w$, which is a function of the decision variables, i.e., generation, arbitrage and transmission levels, of all players in the lower 
level problem. $P_{i^{*} t w}($.$) is a probabilistic function because of the$ stochastic intermittent generation in our model.

Given that the wind and storage technologies have unequal lifespans, we compare their equivalent annual unit costs and consider the equivalent annual budget in our model. Considering the relation between the unit investment cost, $I$, and the equivalent annual unit cost, $\bar{I}$, for a technology with lifespan of PL, that is, $I=\sum_{y=1}^{\mathrm{PL}} \frac{\bar{I}}{(1+r)^{y}}$, the equivalent annual unit costs of wind and storage technologies, $\overline{I^{\mathrm{ig}}}$ and $\overline{I^{\mathrm{s}}}$, become as [23]:

$$
\begin{aligned}
& \overline{I^{\mathrm{ig}}}=\frac{r I^{\mathrm{ig}}}{1-(1+r)^{-\mathrm{PL}^{\mathrm{ig}}}} \\
& \overline{I^{\mathrm{s}}}=\frac{r I^{\mathrm{s}}}{1-(1+r)^{-\mathrm{PL}^{\mathrm{s}}}}
\end{aligned}
$$

where $I^{\mathrm{ig}}$ and $I^{\mathrm{s}}$ are the unit investment costs, and $\mathrm{PL}^{\mathrm{ig}}$ and $\mathrm{PL}^{\mathrm{s}}$ are the life spans of wind and storage technologies, respectively. The parameter $r$ represents the discount rate.

Based on the equivalent annual unit costs of wind and storage technologies, $\overline{I^{\mathrm{ig}}}$ and $\overline{I^{\mathrm{s}}}, \overline{I^{\mathrm{s}}} Q_{i^{*}}^{\mathrm{s}, \mathrm{reg}}$ and $\overline{I^{\mathrm{ig}}} Q_{i^{*}}^{\mathrm{ig}, \text { reg }}$ represent the investment share from the equivalent annual budget, $\overline{\mathrm{B}}$, on wind and storage, respectively.

Given the equations for price volatility and average price (1a$1 b)$, which are functions of the strategy of all firms, and the equations for the equivalent annual cost of wind and storage technologies (2a-2b), we define the upper level optimization problem as:

$$
\min _{Q_{i^{*}}^{\text {sig,reg }}, Q_{i^{*}}^{\text {s,reg }}}(1-k) \sqrt{\overline{\operatorname{Var}}\left(\left\{P_{i^{*} t w}\right\}_{t w}\right)}+k \overline{\mathrm{E}}\left(\left\{P_{i^{*} t w}\right\}_{t w}\right)
$$

s.t.

$$
\overline{I^{\mathrm{s}}} Q_{i^{*}}^{\mathrm{s}, \mathrm{reg}}+\overline{\bar{I}^{\mathrm{ig}}} Q_{i^{*}}^{\mathrm{ig}, \text { reg }}=\overline{\mathrm{B}} \$
$$

where $0 \leq k \leq 1$ represents the weighting coefficient, $Q_{i^{*}}^{\text {ig,reg }}$ is the regulated wind generation capacity and $Q_{i^{*}}^{\mathrm{s}, \text { reg }}$ is the regulated storage capacity in state $i^{*} . \overline{\operatorname{Var}}\left(\left\{P_{i^{*} t w}\right\}_{t w}\right)$ is the normalized level of the average of price volatility levels over the horizon $\left\{1, \ldots, N_{T}\right\}$, i.e., normalized level of $\frac{\sum_{t} \operatorname{Var}\left(\left\{P_{i^{*}} t_{w}\right\}_{w}\right)}{N_{T}}$, and $\overline{\mathrm{E}}\left(\left\{P_{i^{*} t w}\right\}_{t w}\right)$ is the normalized level of the average of mean prices over the horizon $\left\{1, \ldots, N_{T}\right\}$, i.e., normalized level of $\frac{\sum_{t} \mathrm{E}\left(\left\{P_{i^{*}}\right\}_{w}\right)}{N_{T}}$. The normalized levels of price volatility and mean price, which are between zero and one, indicate their ratio with respect to their base values, i.e., with respect to their amounts when there is no regulated wind and storage firm in the market.

\section{B. Lower-level Problem}

In the lower level problem, the strategies of all market players and the nodal market prices are obtained by solving a stochastic Cournot-based game between intermittent generators, synchronous generators, storage firms, and transmission firms. Following the standard Cournot game models [24], any player in our model maximizes its objective function given the decision variables of other players. Our game model, which considers different wind and solar power availability scenarios with given probabilities, is consistent with the Bayesian game definition. Players maximize their utility functions over a set of scenarios with a given probability distribution in a Bayesian game [8]. Note that the decision variables in the upper level problem, $Q_{i^{*}}^{\text {ig,reg }}$ and $Q_{i^{*}}^{\mathrm{s}, \mathrm{reg}}$, are the wind and storage capacity amounts of a regulated wind-storage firm in state $i^{*}$.
The market price in state $i$ at time $t$ under scenario $w$ is represented in our model by an exponential inverse demand function [21]:

$$
P_{i t w}\left(y_{i t w}\right)=\alpha_{i t} e^{-\beta_{i t} y_{i t w}}
$$

where $\alpha_{i t}$ and $\beta_{i t}$ are positive real values representing in the price function, and $y_{i t w}$ is the net electricity demand in state $i$ at time $t$ under scenario $w$.

The lower level problem in our bi-level model is developed based on DC Load Flow equations. The equality between electricity supply and demand in each state and at any time, i.e., the the nodal electricity balance, is ensured in our model with the following equations:

$$
\begin{array}{r}
y_{i t w}=\sum_{m \in \mathcal{N}_{i}^{\mathrm{ig}}} q_{m i t w}^{\mathrm{ig}}+\sum_{n \in \mathcal{N}_{i}^{\mathrm{sg}}} q_{n i t w}^{\mathrm{sg}}+\sum_{b \in \mathcal{N}_{i}^{\mathrm{s}}} q_{b i t w}^{\mathrm{s}}+ \\
\sum_{j \in \mathcal{N}_{i}^{\mathrm{tr}}} q_{i j t w}^{\mathrm{tr}} \forall i \neq i^{*} \\
y_{i t w}=\sum_{m \in \mathcal{N}_{i}^{\mathrm{ig}}} q_{m i t w}^{\mathrm{ig}}+\sum_{n \in \mathcal{N}_{i}^{\mathrm{sg}}} q_{n i t w}^{\mathrm{sg}}+\sum_{b \in \mathcal{N}_{i}^{\mathrm{s}}} q_{b i t w}^{\mathrm{s}}+ \\
\sum_{j \in \mathcal{N}_{i}^{\mathrm{tr}}} q_{i j t w}^{\mathrm{tr}}+q_{i t w}^{\mathrm{ig}, \mathrm{reg}}+q_{i t w}^{\mathrm{s}, \mathrm{reg}} i=i^{*}
\end{array}
$$

where $q_{m i t w}^{\mathrm{ig}}$ is the generation strategy of the $m$ th intermittent generator located in state $i, q_{n i t w}^{\mathrm{sg}}$ is the generation strategy of the $n$th synchronous generator located in state $i, q_{b i t w}^{\mathrm{s}}$ is the charge/discharge strategy of the storage firm $b$ located in state $i, q_{i j t w}^{\mathrm{tr}}$ is the transmission strategy of line between states $i$ and $j$, and $q_{i t w}^{\mathrm{ig}, \mathrm{reg}}$ and $q_{i t w}^{\mathrm{s}, \mathrm{reg}}$ are the wind generation strategy and the storage charge/discharge strategy of the regulated firm in state $i^{*}$, respectively, at time $t$ and under scenario $w$.

In what follows, we use $P_{i t w}($.$) to refer to the market price in$ (4).

1) Intermittent Generators: The $m$ th intermittent generator (wind or solar) in state $i$ determines its best response strategy by solving the following profit maximization problem:

$$
\begin{aligned}
& \underset{\left\{q_{m i t w}^{\mathrm{ig}}\right\}_{t w} \succeq 0}{\max } \sum_{w=1}^{N_{W}} \Psi_{w} \sum_{t=1}^{N_{\mathrm{T}}}\left(P_{i t w}(.)-c_{m i}^{\mathrm{ig}}\right) q_{m i t w}^{\mathrm{ig}} \\
& \text { s.t. } \\
& q_{m i t w}^{\mathrm{ig}} \leq \omega_{i t w} Q_{m i}^{\mathrm{ig}} \quad \forall t, w \\
& P_{i t w}(.) \leq P^{\text {cap }} \quad \forall t, w
\end{aligned}
$$

where $q_{m i t w}^{\mathrm{ig}}$ (decision variable) is the generation level of the intermittent generator $m$ in state $i$ at time $t$ under scenario $w, Q_{m i}^{\text {ig }}$ is its maximum generation capacity, and $c_{m i}^{\mathrm{ig}}$ is its marginal cost of generation. The constraint (6b) limits the electricity generation to the available generation capacity of the firm, considering the energy availability coefficient $\omega_{i t w}$ in state $i$ at time $t$ under scenario $w$. The availability coefficient $\omega_{i t w}$ is the source of stochasticity in our model. The constraint (6c) ensures that the market price is always less than the cap price $P^{\text {cap }}$.

2) Synchronous Generators: The best response strategy of the $n$th synchronous generator in state $i$ is obtained by solving the following profit maximization problem:

$$
\max _{\left\{q_{n i t w}^{\mathrm{sg}}\right\}_{t w} \succeq 0} \sum_{w=1}^{N_{W}} \Psi_{w} \sum_{t=1}^{N_{\mathrm{T}}}\left(P_{i t w}(.)-c_{n i}^{\mathrm{sg}}\right) q_{n i t w}^{\mathrm{sg}}
$$




$$
\begin{aligned}
& \text { s.t. } \\
& q_{n i t w}^{\mathrm{sg}} \leq Q_{n i}^{\mathrm{sg}} \quad \forall t, w \\
& q_{n i t w}^{\mathrm{sg}}-q_{n i(t-1) w}^{\mathrm{sg}} \leq R_{n i}^{\mathrm{up}} Q_{n i}^{\mathrm{sg}} \quad \forall t, w \\
& q_{n i(t-1) w}^{\mathrm{sg}}-q_{n i t w}^{\mathrm{sg}} \leq R_{n i}^{\mathrm{dn}} Q_{n i}^{\mathrm{sg}} \quad \forall t, w \\
& \sum_{t} q_{n i t w}^{\mathrm{sg}} \leq G_{n i} \quad \forall w \\
& P_{i t w}(.) \leq P^{\mathrm{cap}} \quad \forall t, w
\end{aligned}
$$

where $q_{\text {nitw }}^{\mathrm{sg}}$ (decision variable) is the generation level of the synchronous generator $n$ in state $i$ at time $t$ under scenario $w$, $Q_{n i}^{\mathrm{sg}}$ is its generation capacity, and $c_{n i}^{\mathrm{sg}}$ is its marginal cost of generation. The constraint ( $7 b)$ considers the maximum capacity limit and the constraints $(7 \mathrm{c}-7 \mathrm{~d})$ consider the ramping up and down limits, $R_{n i}^{\mathrm{up}}$ and $R_{n i}^{\mathrm{dn}}$, respectively. The constraint (7e) considers the inter-temporal energy availability $G_{n i}$, e.g., the total hydro power generation over a year due to the dam water availability during that period.

3) Storage Firms: The best response strategy of the $b$ th storage firm in state $i$ is the solution of the following profit maximization problem:

$$
\left.\max _{\substack{\left\{q_{b i t w}^{\mathrm{dis}}, q_{b i t w}^{\mathrm{ch}} \\\left\{q_{b i t w}^{\mathrm{s}}\right\}_{t w}\right.}}\right\}_{t w} \sum_{w=1}^{N_{W}} \Psi_{w} \sum_{t=1}^{N_{\mathrm{T}}} P_{i t w}(.) q_{b i t w}^{\mathrm{s}}-c_{b i}^{\mathrm{s}}\left(q_{b i t w}^{\mathrm{dis}}+q_{b i t w}^{\mathrm{ch}}\right)
$$

s.t.

$$
\begin{aligned}
& q_{b i t w}^{\mathrm{s}}=\eta_{b i}^{\mathrm{dis}} q_{b i t w}^{\mathrm{dis}}-\frac{q_{b i t w}^{\mathrm{ch}}}{\eta_{b i}^{\mathrm{ch}}} \quad \forall t, w \\
& q_{b i t w}^{\mathrm{dis}} \leq \zeta_{b i}^{\mathrm{dis}} Q_{b i}^{\mathrm{s}} \quad \forall t, w \\
& q_{b i t w}^{\mathrm{ch}} \leq \zeta_{b i}^{\mathrm{ch}} Q_{b i}^{\mathrm{s}} \quad \forall t, w \\
& 0 \leq \sum_{k=1}^{t}\left(q_{b i k w}^{\mathrm{ch}}-q_{b i k w}^{\mathrm{dis}}\right) \Delta \leq Q_{b i}^{\mathrm{s}} \quad \forall t, w \\
& P_{i t w}(.) \leq P^{\text {cap }} \quad \forall t, w
\end{aligned}
$$

where $q_{b i t w}^{\mathrm{ch}}$ and $q_{b i t w}^{\mathrm{dis}}$ (decision variables) are the charge and discharge levels of the storage firm $b$ in state $i$ at time $t$ under scenario $w$, respectively, $q_{b i t w}^{\mathrm{s}}$ (intermediate decision variable) is the net charge/discharge level, $c_{b i}^{\mathrm{s}}$ is the marginal cost of charge/discharge, and $\eta_{b i}^{\text {ch }}$ and $\eta_{b i}^{\text {dis }}$ are the charging and discharging efficiencies, respectively. The equality $(8 \mathrm{~b})$ indicates the net outflow or inflow of electricity, the constraints (8c) and (8d) limit the output/input energy flow of the firm, with coefficients $\zeta_{b i}^{\text {dis }}$ and $\zeta_{b i}^{\mathrm{ch}}$, respectively. The parameters $\zeta_{b i}^{\mathrm{ch}}$ and $\zeta_{b i}^{\text {dis }}$ indicate the percentage of the storage capacity $Q_{b i}^{\mathrm{s}}$ that can be charged or discharged during time period $\Delta$, which are considered equal to $10 \%$ per hour for pump-hydro and $50 \%$ per hour for battery in our study. The constraint (8e) limits the total stored energy to its maximum capacity, assuming that the storage devices are initially fully discharged.

4) Transmission Firms: The best response strategy of the transmission line (interconnector) between states $i$ and $j$ is obtained by solving the following profit maximization problem:

$$
\begin{aligned}
\left.\max _{\left\{q_{j i t w}^{\mathrm{tr}}, q_{i j t w}^{\mathrm{tr}}\right.}\right\}_{t w} & \sum_{w=1}^{N_{W}} \Psi_{w} \sum_{t=1}^{N_{\mathrm{T}}}\left(P_{j t w}(.) q_{j i t w}^{\mathrm{tr}}+P_{i t w}(.) q_{i j t w}^{\mathrm{tr}}\right) \\
& \left(1-\gamma_{i j}^{\mathrm{tr}}\right)+\gamma_{i j}^{\mathrm{tr}}\left(\frac{P_{j t w}(.)}{-\beta_{j t}}+\frac{P_{i t w}(.)}{-\beta_{i t}}\right)
\end{aligned}
$$

$$
\begin{aligned}
& \text { s.t. } \\
& q_{i j t w}^{\mathrm{tr}}=-q_{j i t w}^{\mathrm{tr}} \quad \forall t, w \\
& -Q_{i j}^{\operatorname{tr}} \leq q_{i j t w}^{\mathrm{tr}} \leq Q_{i j}^{\mathrm{tr}} \quad \forall t, w \\
& P_{k t w}(.) \leq P^{\mathrm{cap}} \quad k \in\{i, j\}, \forall t, w
\end{aligned}
$$

where $q_{i j t w}^{\mathrm{tr}}$ (decision variable) is the electricity transmitted from state $j$ to state $i$ at time $t$ under scenario $w$, and $Q_{i j}^{\mathrm{tr}}$ is the capacity of transmission line between states $i$ and $j$. The transmission firm between states $i$ and $j$ is a strategic player when $\gamma_{i j}^{\text {tr }}$ is zero and is a regulated player when $\gamma_{i j}^{\mathrm{tr}}$ is one. It is discussed in [21] that maximizing $P_{j t}(.) q_{j i t w}^{\mathrm{tr}}+P_{i t w}(.) q_{i j t w}^{\mathrm{tr}}$ is equal to maximizing the profit from electricity transmission between states $i$ and $j$. Besides, it can be shown that maximizing $\frac{P_{j t w}(.)}{-\beta_{j t}}+\frac{P_{i t w}(.)}{-\beta_{i t}}$ is equivalent to maximizing the social welfare (the total surplus of consumers and producers) when the transmission firm between states $i$ and $j$ is regulated. Note that the electricity markets with regulated transmission firms are called electricity markets with transmission constraints in the literature, e.g., [25], [26]. The constraint (9b) ensures that electricity does not flow simultaneously in both directions of the line, and the constraint (9c) limits the electricity flow between states $i$ and $j$ to the capacity of the line.

5) State-owned Wind-Storage Firm: The best response strategy of the state-owned wind-storage firm in state $i^{*}$, which is a regulated firm, is determined by solving the following optimization problem:

$$
\begin{aligned}
& \max _{\left\{q_{i^{*} t w}^{\text {ig, reg }}\right\}_{t w} \succeq 0,} \sum_{w=1}^{N_{W}} \Psi_{w} \sum_{t=1}^{N_{\mathrm{T}}} \frac{P_{i^{*} t w}(.)}{-\beta_{i^{*} t}}-c_{i^{*}}^{\mathrm{ig}, \text { reg }} q_{i^{*} t w}^{\mathrm{ig}, \text { reg }} \\
& \left\{q_{i^{*} t w}^{\mathrm{dis}, \mathrm{reg}}, q_{i^{*} t w}^{\mathrm{ch}, \mathrm{reg}}\right\}_{t w} \succeq 0, \\
& \left\{q_{i * t w}^{\mathrm{s}, \mathrm{reg}}\right\}_{t w} \\
& -c_{i^{*}}^{\mathrm{s}, \mathrm{reg}}\left(q_{i^{*} t w}^{\mathrm{dis}, \mathrm{reg}}+q_{i^{*} t w}^{\mathrm{ch}, \mathrm{reg}}\right) \\
& \text { s.t. } \\
& q_{i^{*} t w}^{\mathrm{ig}, \mathrm{reg}} \leq \omega_{i^{*} t w} Q_{i^{*}}^{\mathrm{ig}, \mathrm{reg}} \quad \forall t, w \\
& q_{i^{*} t w}^{\text {s,reg }}=\eta_{i^{*}}^{\text {dis,reg }} q_{i^{*} t w}^{\text {dis,reg }}-\frac{q_{i^{*} t w}^{\text {ch,reg }}}{\eta_{i^{*}}^{\text {ch,reg }}} \quad \forall t, w \\
& q_{i^{*} t w}^{\text {dis,reg }} \leq \zeta_{i^{*}}^{\text {dis,reg }} Q_{i^{*}}^{\mathrm{s}, \text { reg }} \quad \forall t, w \\
& q_{i^{*} t w}^{\mathrm{ch}, \mathrm{reg}} \leq \zeta_{i^{*}}^{\mathrm{ch}, \mathrm{reg}} Q_{i^{*}}^{\mathrm{s}, \mathrm{reg}} \quad \forall t, w \\
& 0 \leq \sum_{k=1}^{t}\left(q_{i^{*} k w}^{\text {ch,reg }}-q_{i^{*} k w}^{\text {dis,reg }}\right) \Delta \leq Q_{i^{*}}^{\mathrm{s}, \text { reg }} \quad \forall t, w \\
& P_{i^{*} t w}(.) \leq P^{\text {cap }} \quad \forall t, w
\end{aligned}
$$

where $q_{i^{*} t w}^{\mathrm{ig}, \mathrm{reg}}$ (decision variable) is the wind (intermittent) generation level of the regulated firm in state $i^{*}$ at time $t$ under scenario $w, Q_{i^{*}}^{\mathrm{ig}, \mathrm{reg}}$ is its maximum wind generation capacity, and $c_{i^{*}}^{\mathrm{ig}, \mathrm{reg}}$ is its marginal cost of wind generation. Moreover, $q_{i^{*} t w}^{\mathrm{ch}, \mathrm{reg}}, q_{i^{*} t w}^{\mathrm{dis}, \mathrm{reg}}$ (decision variables), and $q_{i^{*} t w}^{\mathrm{s}, \mathrm{reg}}$ (intermediate decision variable) are the charge, discharge and net charge/discharge levels of the regulated firm in state $i^{*}$ at time $t$ under scenario $w$, respectively. The constraint (10b) is similar to the constraint in the wind generation problem (6b), and the constraints (10c)-(10f) are similar to the constraints in the storage arbitrage problem (8b)(8e). It can be shown that maximizing the $\frac{P_{i^{*} t w}(.)}{-\beta_{i^{*} t}}-c_{i^{*}}^{\text {ig, reg }} q_{i^{*} t w}^{\text {ig, reg }}-$ $c_{i^{*}}^{\mathrm{s}, \mathrm{reg}}\left(q_{i^{*} t w}^{\mathrm{dis}, \mathrm{reg}}+q_{i^{*} t w}^{\mathrm{ch}, \mathrm{reg}}\right)$ is equivalent to maximizing the social welfare for the regulated wind-storage firm. 


\section{SOlution ApPROACH}

Here, the bi-level storage and wind allocation problem reducing the average price and price volatility is transformed into a single-level Mathematical Problem with Equilibrium Constraints (MPEC).

\section{A. Solution Method for the lower level problem}

The regulated wind and storage capacities are the only variables that couple the scenarios in the lower level problem. Therefore, for any regulated wind and storage capacity amounts, each scenario of the lower level problem can be solved autonomously and the market equilibrium can be obtained by solving the KKT equations of all firms. The existence of the Bayes-NE solution at the lower level problem is stated in Proposition 1.

Proposition 1: For any vector of regulated wind and storage capacity amounts, $\left[Q_{i^{*}}^{\mathrm{ig}, \text { reg }}, Q_{i^{*}}^{\mathrm{s} \text {,reg }}\right]$, the lower level game admits a Bayes-NE.

Proof: The objective function of any firm in the game is continuous and quasi-concave in its strategy, and their strategy space is non-empty, compact and convex. Therefore, according to Theorem 1.2 in [27], the lower level game admits a BayesNE.

In the lower level problem, the nodal market prices depend on the regulated wind and storage capacities through the constraints (10b) and (10d-10f). This dependency allows us to minimize the objective function on the upper level problem using the optimal values of regulated wind and storage capacities.

\section{B. Solution Method for the equivalent single level problem}

Imposing the KKT conditions of all firms as constraints in the optimization problem (3), we can transform our bi-level problem into the following single-level optimization problem:

$$
\begin{aligned}
& \min (1-k) \sqrt{\overline{\operatorname{Var}}\left(\left\{P_{i^{*} t w}\right\}_{t w}\right)}+k \overline{\mathrm{E}}\left(\left\{P_{i^{*} t w}\right\}_{t w}\right) \\
& \text { s.t. }
\end{aligned}
$$

$$
\begin{aligned}
& (3 b) \\
& \operatorname{KKT}(6 a-6 c) \\
& \operatorname{KKT}(7 a-7 f) \\
& \operatorname{KKT}(8 a-8 f) \\
& \operatorname{KKT}(9 a-9 d) \\
& \operatorname{KKT}(10 a-10 g)
\end{aligned}
$$

where the optimization variables are the regulated wind and storage capacities, the bidding strategies of all firms, and the set of all Lagrangian multipliers. Note that the feasible region is not necessarily convex or even connected because of the nonlinear complementary constraints. It is possible to write the equivalent single level problem (11) as a Mixed-Integer Non-Linear Problem (MINLP), but the large number of integer variables makes the problem computationally infeasible.

Considering the equality constraint ( $3 b$ ), there is just one decision variable on the upper level problem. We perform a uniform line search on the variable $Q_{i^{*}}^{\mathrm{ig}, \text { reg }}$, i.e., the single decision variable of the upper level problem, with $N$ iterations. We increase the regulated wind capacity by $\Delta Q^{\mathrm{ig}, \text { reg }}$ and decrease the regulated storage capacity by $\Delta Q^{\mathrm{s}, \mathrm{reg}}$, which is a function of $\Delta Q^{\mathrm{ig} \text {,reg }}$, and find the Bayes-NE solution of the lower level game at each iteration. Comparing the average price and price volatility calculated at different iterations, we find the optimal regulated wind and storage allocation, as described in Algorithm 1.

Algorithm 1 The line search ( $N$-step) algorithm for finding the wind-storage allocation.

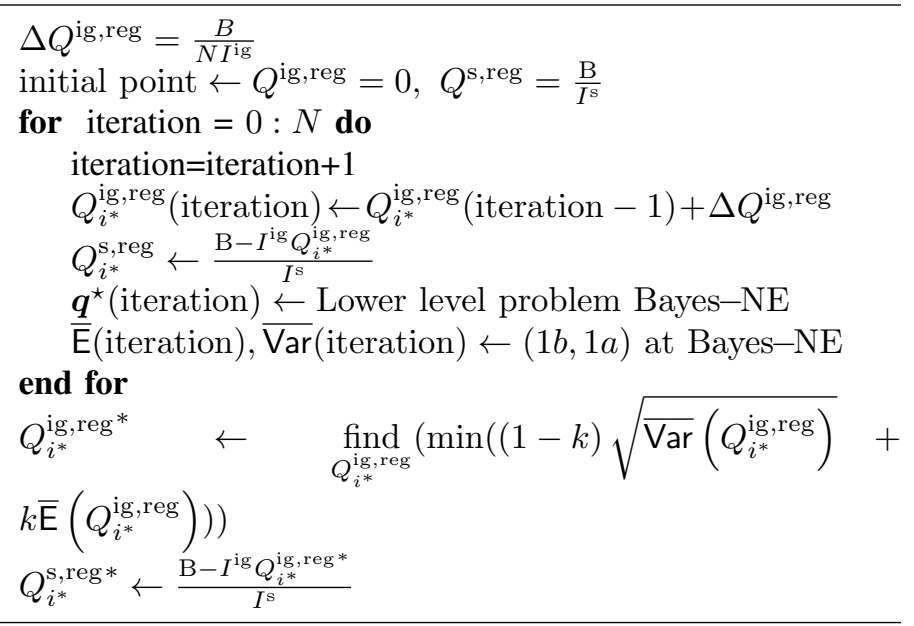

\section{Case Study and Simulation Results}

In this section, we apply our bi-level price management framework to Australia's National Electricity Market (NEM). NEM has a regional pricing mechanism, which sets the marginal value of demand at each state as the regional price, in five states of South Australia (SA), Queensland (QLD), Tasmania (TAS), Victoria (VIC) and New South Wales (NSW). The inverse demand functions in our model are calibrated with historical demand and price data from the year 2016. Different types of electricity generation firms, such as coal, gas, hydro, biomass, and wind, with total generation capacity of $46 \mathrm{GW}$ were active in NEM in 2016 [28]. In our numerical study, we consider 365 scenarios each representing a 24-hour wind power availability and electricity demand profiles. The realistic data in different regions of NEM from the year 2016 is used to generate the scenario set (Source of data: AEMO). Note that all the prices are in Australian dollar.

\section{A. Impact of Generation Capacity, Gas Price and Transmission Line on Average Price and Price Volatility in NEM}

In this subsection, we first study the average price and price volatility in the NEM by considering two cases. In our primary case, the NEM market is simulated based on the available data in 2016. In our secondary case, the Hazelwood coal power plant in VIC is closed down [29], the gas price in total NEM is increased, and the Basslink transmission line, between VIC and TAS, which was under maintenance in 2016, is restarted in comparison to the primary case. Table I compares the simulated wholesale electricity prices in five regions of NEM in the primary and secondary cases. Our simulation results show that the average price of electricity increases in all regions, about $14.27 \%$ in NEM, due to Hazelwood power plant closure and gas price surge. The highest rate of price increment belongs to VIC, about $40.33 \%$, where the coal plant was located, following by its neighboring region SA with $19.80 \%$. According to our numerical results, restarting the Basslink interconnector between VIC and TAS reduces the impacts of coal plant closure and gas price surge on the electricity price in TAS, which increases just by $3.58 \%$ in average.

Our calculation also shows that price volatility increases in NEM after the coal plant closure and gas price surge. The square root of price volatility increases by $17.7 \%$ in NEM, where VIC 
TABLE I: Wholesale electricity prices $(\$ / M W h)$ in five-state NEM market in primary and secondary cases.

\begin{tabular}{llllcll}
\hline \hline & SA & QLD & TAS & VIC & NSW & NEM \\
\hline Primary Case & 108.97 & 72.32 & 99.64 & $\mathbf{5 7 . 4 8}$ & 58.81 & 67.27 \\
Secondary Case & 130.55 & 78.94 & 103.20 & $\mathbf{8 0 . 6 6}$ & 61.65 & 76.87 \\
Change\% & 19.80 & 9.16 & 3.58 & $\mathbf{4 0 . 3 3}$ & 4.83 & 14.27 \\
\hline \hline
\end{tabular}

experiences the highest increase rate of $41.5 \%$. The Basslink transmission line also suppresses the price volatility in TAS due to the Hazelwood closure and gas price surge.

\section{B. Managing the Average Price and Price Volatility by Only Regulated Wind or Only Regulated Storage}

In this subsection, we study the impact of installing only regulated wind or only regulated storage on the average price and price volatility in VIC, where the coal power plant is closed down. We start our simulations with the equivalent annual budget of 300 $\mathrm{m} \$$, and perform the sensitivity analysis with other amounts of the equivalent annual budget, between zero and $300 \mathrm{~m} \$$, later. Considering the investment cost of $2400 \$ / \mathrm{kW}$ and lifespan of 25 years, the equivalent annual unit cost is $96 \$ /(\mathrm{kW} . \mathrm{yr})$ for wind generation. Also, with the investment cost of $600 \$ / \mathrm{kWh}$ and lifespan of 10 years, the equivalent annual unit cost is 60 $\$ /(\mathrm{kWh} . y r)$ for battery storage. Therefore, the equivalent annual budget of $300 \mathrm{~m} \$$ is almost equivalent to $3125 \mathrm{MW}$ wind capacity or $5000 \mathrm{MWh}$ battery capacity.

Fig. 1 shows the impact of installing only 3125 MW regulated wind on the wholesale electricity prices in VIC. The regulated wind in our model with capacity of $3125 \mathrm{MW}$ generates electricity with average level of $975 \mathrm{MW}$, i.e., with capacity factor of 31\%, in VIC. The generation of the regulated wind firm results in the average peak and off-peak wholesale price reductions of $28 \$ / M W h$ and 5 \$/MWh, respectively, in VIC. The average wholesale electricity price in VIC decreases from 80.6 \$/MWh to $62 \$ / \mathrm{MWh}$ due to the $3125 \mathrm{MW}$ wind capacity addition.

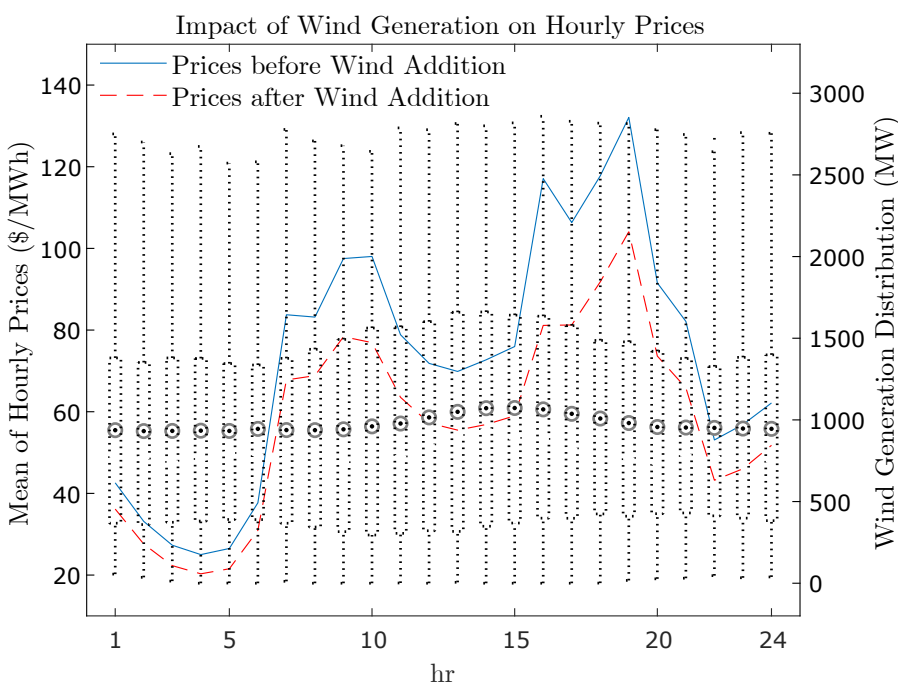

Fig. 1: Mean (over 365 scenarios) wholesale electricity prices in VIC before and after addition of only 3125 MW regulated wind generation capacity (the central marks show the mean levels and the bottom and top edges of the boxes indicate the 25th and 75th percentiles of wind generation).

Fig. 2 shows the impact of installing only 5000 MWh regulated battery on the mean wholesale electricity prices in VIC. According to this figure, the regulated battery in VIC makes profit from electricity arbitrage, i.e., charges at off-peak times, with average peak charge level of $1271 \mathrm{MW}$, and discharges at peak hours, with average peak discharge level of $1022 \mathrm{MW}$. The charge/discharge of the installed battery approximately results in the average peak price reduction of $47 \$ / \mathrm{MWh}$ and the average off-peak price increment of $16 \$ / \mathrm{MWh}$ in VIC. The average wholesale electricity price in VIC decreases from $80.6 \$ / \mathrm{MWh}$ to $72.9 \$ / \mathrm{MWh}$ due to the addition of $5000 \mathrm{MWh}$ regulated battery. This observation confirms that wind power generators are more efficient in average price reduction than storage firms.

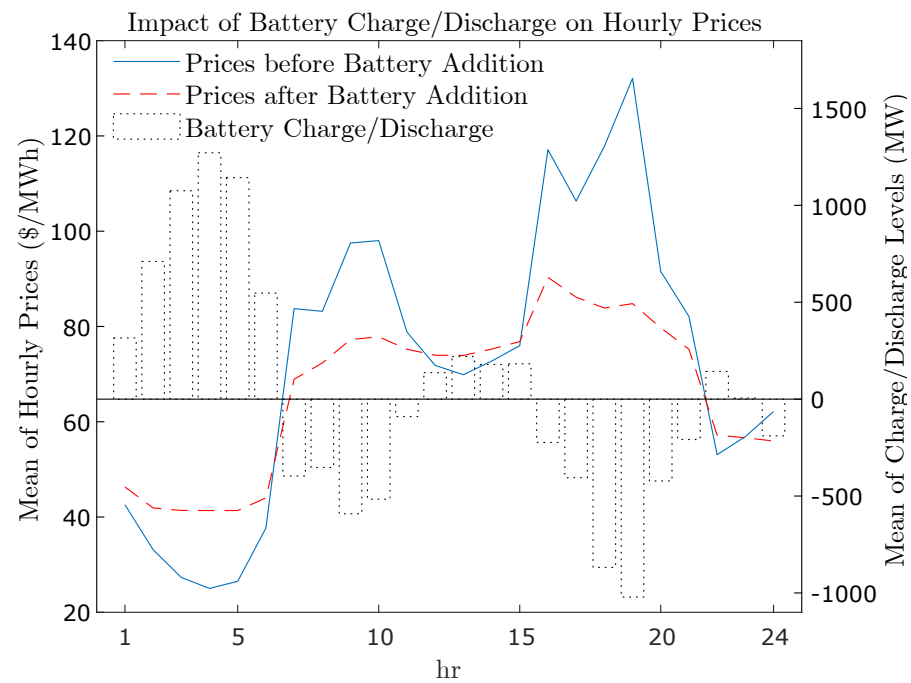

Fig. 2: Mean (over 365 scenarios) wholesale electricity prices in VIC before and after addition of only $5000 \mathrm{MWh}$ regulated battery storage capacity.

Fig. 3a and $3 b$ compare the impact of a regulated wind with that of a regulated storage on the average price and the price volatility, respectively, in VIC when the equivalent annual budget increases from zero to $300 \mathrm{~m} \$$. It can be seen that for different levels of budget, i.e., different levels of capacity, the regulated storage is more efficient in reducing the price volatility whereas the regulated wind is more efficient in reducing the average price. Given the equivalent annual budget of $300 \mathrm{~m} \$$, the regulated storage and the regulated wind reduce the square root of price volatility in VIC by $71.14 \%$ and $53.55 \%$, respectively. However, with the same equivalent annual budget, regularized storage and wind firms reduce the average price in VIC by $10.04 \%$ and $29.08 \%$, respectively. This observation quantitatively shows the effectiveness of storage in price volatility reduction and wind in average price reduction.

Moreover, in addition to mean price and price volatility reduction impacts, the cost analysis of the regulated wind and regulated storage can affect the investment decisions. Fig. 3c indicates the cost analysis of the regulated wind and regulated storage in VIC when the equivalent annual budget varies from zero to $300 \mathrm{~m} \$$. The life-time rate of return less than $100 \%$ shows a financially unprofitable investment [23]. Based on this figure, the regulated wind is financially profitable in VIC when the equivalent annual investment cost is less than $300 \mathrm{~m} \$$, but the regulated storage makes profit in VIC when the equivalent annual investment cost is less than $100 \mathrm{~m} \$$. Thus, the (low) life time rate of return of storage firms further reduces the desirability of storage only solution for the market intervention. Note that future reduction in battery cost 
makes the large investments on batteries profitable.

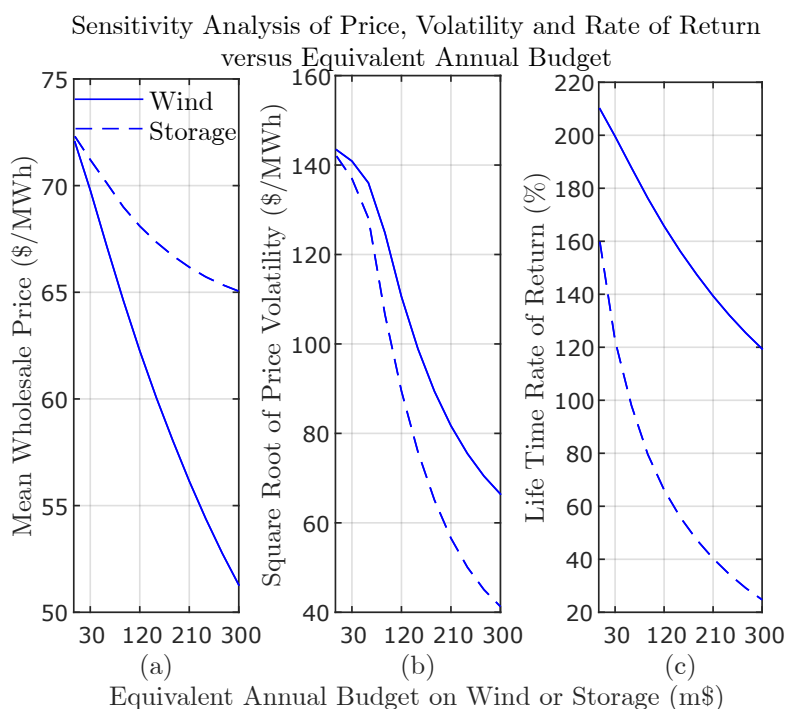

Fig. 3: The mean price, the square root of price volatility, and the life time rate of return for only regulated wind and only regulated battery allocation versus the equivalent annual budget in VIC.

\section{Managing the Average Price and Price Volatility by Mixture of Regulated Wind and Storage in VIC}

In this subsection, we study the impact of jointly optimal regulated wind and storage allocation on the mean price and price volatility. Fig. 4 illustrates the normalized mean wholesale price as well as the normalized square roof of price volatility for different mixtures of wind and battery allocation with the equivalent annual budget of $300 \mathrm{~m} \$$ in VIC. The mean wholesale prices are normalized with base value of $73 \$ / \mathrm{MWh}$, which is the average price in the market before adding regulated wind-storage capacities, and the square root of price volatilities are normalized with the base value of $143 \$ / \mathrm{MWh}$, which is the square root of price volatility in the market before adding regulated wind-storage capacities. According to Fig. 4, the increase of the regulated wind share, $\xi$, (or equivalently, the decrease of regulated storage share, $1-\xi)$ results in comparatively lower average prices but higher price volatility levels in the market, and vice versa. Therefore, depending on the importance of average price or price volatility, i.e., the coefficient $k$, the total budget can be allocated on a mixture of regulated wind and battery capacities.

Fig. 5 shows the budget allocation share between regulated wind $(\xi)$ and regulated battery $(1-\xi)$ when the weighting coefficient of price volatility and average price in the upper level problem (3), $k$, varies from zero to one. The logistic shape of the optimal budget share function with respect to the weighting coefficient $k$ verifies our observations regarding the impacts of wind and storage firms on the price. The optimal share of regulated wind is more than that of the regulated storage when average price reduction is prioritized, i.e., when $0.5 \leq k \leq 1$. Similarly, when price volatility reduction is more important, i.e., when $0 \leq k \leq 0.5$, the optimal share of regulated battery is more than that of the regulated wind. The decision making on the budget share is highly sensitive with respect to parameter $k$ when the average price and the price volatility are almost equally important, i.e., $0.4 \leq k \leq 0.6$.

Moreover, the annotated data in Fig. 5 is provided to compare the Life-time Rate of Return (LRR), the change of consumer

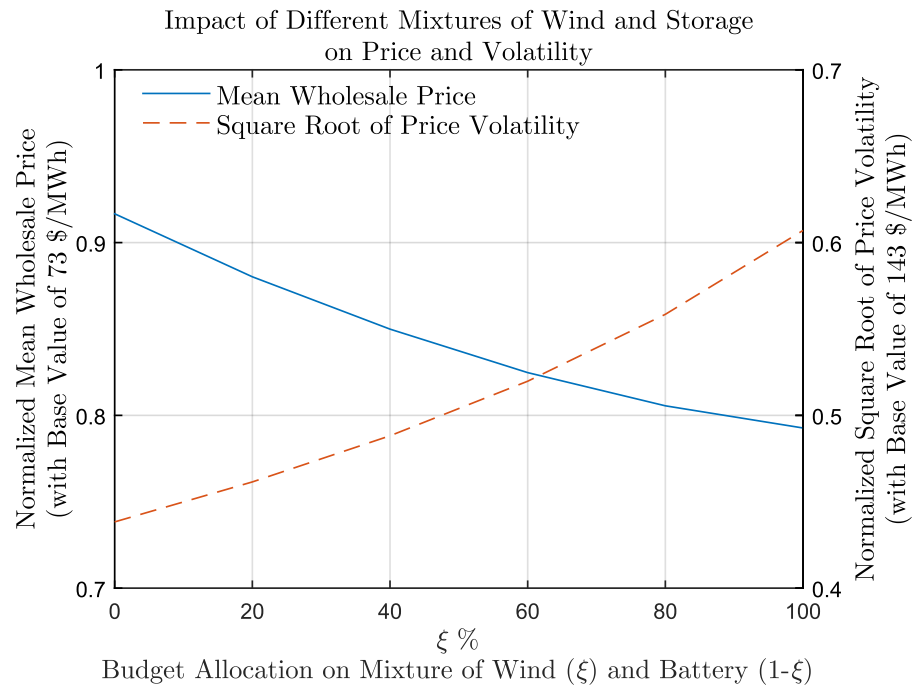

Fig. 4: Normalized mean wholesale price and square root of price volatility for different mixtures of regulated wind and regulated battery with the equivalent annual budget of $300 \mathrm{~m} \$$ in VIC.

surplus or profit $(\Delta \mathrm{cs})$, the normalized amount of average price $\left(\mathrm{P}_{\text {Mean }}\right)$, and the normalized amount of square root of price volatility $\left(\mathrm{P}_{\mathrm{SD}}\right)$ when different mixtures of wind and battery are installed in VIC, given the equivalent annual budget of $300 \mathrm{~m} \$$. Investing all the budget on battery, we can achieve the minimum square root of price volatility $62.7 \$ / \mathrm{MWh}(43.8 \%$ of its base value), while investing all the budget on wind, we can get the minimum average price $57.5 \$ \mathrm{MWh}$ (79.2\% of its base value) and the highest life-time rate of return $122 \%$. Investing the budget on regulated wind is financially more profitable than on regulated battery. It is required to allocate at least $60 \%$ of the equivalent annual budget on wind to make the investment on any mixture of wind-storage financially economical, i.e., having the life-time rate of return above $100 \%$. Lastly, the consumer surplus (profit) increases between $0.93 \%$ and $1.69 \%$ when the regulated windstorage firm is considered in the market. It can be seen that the mixtures of regulated wind and storage capacities are more effective than storage-only solution in increasing the consumer surplus.

\section{CONCLUSION}

Closure of base-load coal power plants, and gas price surge may increase the average price and price volatility in electricity markets. Our study presents an optimization framework which allocates a budget on regulated wind and storage capacities in order to minimize the weighted sum of the average price and the price volatility. Based on our numerical results in NEM, the impacts of regulated wind and storage on average price and price volatility can be summarized as:

- Both storage and wind affect the average price and price volatility in electricity markets. Storage technologies can reduce the price and the price volatility by electricity price arbitrage. Being spread across the network, wind turbines can also decrease the price and volatility in electricity markets. In our model, a single node represents an entire state, and hence, incorporates diversity of wind generation across a large geographic region that counteracts natural intermittency of wind generation.

- Given the equivalent annual budget of $300 \mathrm{~m} \$$ to invest on regulated wind-storage capacities in VIC, storage is comparatively $47.8 \%$ more efficient in price volatility reduction than 
Optimal Share of Budget on Wind and Storage versus the Weighting Coefficient $k$

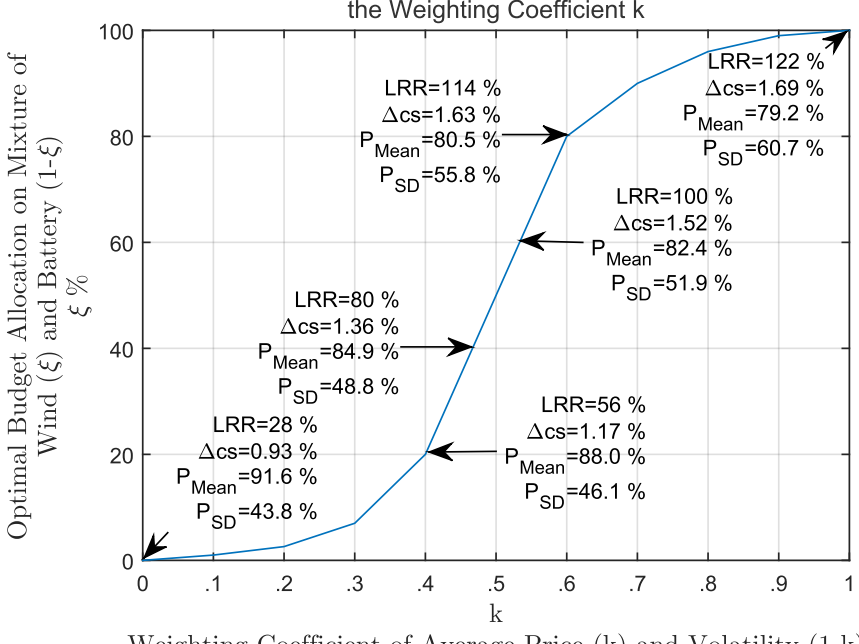

Weighting Coefficient of Average Price (k) and Volatility (1-k)

Fig. 5: The budget allocation share between the regulated wind and the regulated battery as a function of the weighting factor $k$ with the equivalent annual budget of $300 \mathrm{~m} \$$ in VIC. Lifetime Rate of Return (LRR), change of consumer surplus or profit $(\Delta c s)$, normalized amount of average price $\left(\mathrm{P}_{\text {Mean }}\right)$, and normalized amount of square root of price volatility $\left(\mathrm{P}_{\mathrm{SD}}\right)$ are annotated.

wind whereas wind is $13.5 \%$ more efficient in average price reduction. Based on the importance of average price and price volatility, a mixture of regulated wind and storage capacities can be allocated in a region to reach the desired level of price and volatility in the market.

- When investing on only storage is not economical, the regulated firm can make profit by investing on a mixture of wind and storage capacities. Minimum $60 \%$ budget allocation on wind, given the equivalent annual budget of $300 \mathrm{~m} \$$ in VIC, makes the investment on any mixture of wind/storage economical.

- The mixtures of regulated wind and storage capacities are comparatively more efficient, up to $81.7 \%$ in VIC given the equivalent annual budget of $300 \mathrm{~m} \$$, than the storage-only solution in increasing the consumer surplus (profit) in our study.

- Wind turbine, with small or large capacity, is already a competent technology which is able to recover its life time cost in the market, but storage technology is economical just in small to medium size. However, the mixture of wind and storage capacities, which can optimally reduce the average price and price volatility, may be competent to make profit in the market. Future technology cost reduction can also make it economical to install larger batteries in the market.

Updating our solution approach, we intend to modify our model to decide on the location of wind-storage systems in addition to their capacities and study the impact of wind-storage allocation at different regions on regional market prices in our future work.

\section{REFERENCES}

[1] "The Health of the National Electricity Market," Annual Report Energy Security Board, Tech. Rep., 2017.

[2] D. Chattopadhyay and T. Alpcan, "A Game-Theoretic Analysis of Wind Generation Variability on Electricity Markets," Power Systems, IEEE Transactions on, vol. 29, no. 5, pp. 2069-2077, Sept 2014.

[3] H. Higgs and A. Worthington, "Stochastic price modeling of high volatility, mean-reverting, spike-prone commodities: The Australian wholesale spot electricity market," Energy Economics, vol. 30, p. 31723185, 2008.

[4] C. Latimer, "Victorian power bills soar after hazelwood coal plant closure," March 2018. [Online]. Available: www.theage.com.au
[5] S. Borenstein, "Understanding competitive pricing and market power in wholesale electricity markets," The Electricity Journal, vol. 13, no. 6, pp. $49-57,2000$.

[6] D. McConnell, "A month in, teslas sa battery is surpassing expectations," January 2018. [Online]. Available: http://theconversation.com

[7] D. Wozabal, C. Graf, and D. Hirschmann, "The effect of intermittent renewables on the electricity price variance," OR spectrum, pp. 1-23, 2014.

[8] J. C. Harsanyi, "Games with Incomplete Information Played by "Bayesian" Players, I-III. Part II. Bayesian Equilibrium Points," Management Science, vol. 14 , no. 5 , pp. $320-334,1968$.

[9] N. Li, C. Ukun, E. M. Constantinescu, J. R. Birge, K. W. Hedman, and A. Botterud, "Flexible Operation of Batteries in Power System Scheduling With Renewable Energy," IEEE Transactions on Sustainable Energy, vol. 7, no. 2, pp. 685-696, April 2016.

[10] A. Berrada and K. Loudiyi, "Operation, sizing, and economic evaluation of storage for solar and wind power plants " Renewable and Sustainable Energy Reviews, vol. 59, pp. 1117 - 1129, 2016.

[11] V. Krishnan and T. Das, "Optimal allocation of energy storage in a cooptimized electricity market: Benefits assessment and deriving indicators for economic storage ventures " Energy, vol. 81, pp. 175 - 188, 2015.

[12] M. Sedghi, A. Ahmadian, and M. Aliakbar-Golkar, "Optimal Storage Planning in Active Distribution Network Considering Uncertainty of Wind Power Distributed Generation," IEEE Transactions on Power Systems, vol. 31, no. 1, pp. 304-316, Jan 2016.

[13] L. Zheng, W. Hu, Q. Lu, and Y. Min, "Optimal energy storage system allocation and operation for improving wind power penetration," IET Generation, Transmission Distribution, vol. 9, no. 16, pp. 2672-2678, 2015.

[14] J. Xiao, Z. Zhang, L. Bai, and H. Liang, "Determination of the optimal installation site and capacity of battery energy storage system in distribution network integrated with distributed generation," IET Generation, Transmission Distribution, vol. 10, no. 3, pp. 601-607, 2016.

[15] R. Walawalkar, J. Apt, and R. Mancini, "Economics of electric energy storage for energy arbitrage and regulation in New York ," Energy Policy, vol. 35 , no. 4 , pp. $2558-2568,2007$.

[16] H. Mohsenian-Rad, "Optimal Bidding, Scheduling, and Deployment of Battery Systems in California Day-Ahead Energy Market," IEEE Transactions on Power Systems, vol. 31, no. 1, pp. 442-453, Jan 2016.

[17] H. Akhavan-Hejazi and H. Mohsenian-Rad, "A stochastic programming framework for optimal storage bidding in energy and reserve markets," in Innovative Smart Grid Technologies (ISGT), 2013 IEEE PES, Feb 2013, pp. $1-6$.

[18] H. Mohsenian-Rad, "Coordinated Price-Maker Operation of Large Energy Storage Units in Nodal Energy Markets," IEEE Transactions on Power Systems, vol. 31, no. 1, pp. 786-797, Jan 2016.

[19] E. Nasrolahpour, S. J. Kazempour, H. Zareipour, and W. D. Rosehart, "Strategic Sizing of Energy Storage Facilities in Electricity Markets," IEEE Transactions on Sustainable Energy, vol. 7, no. 4, pp. 1462-1472, Oct 2016.

[20] W.-P. Schill, C. Kemfert et al., "Modeling strategic electricity storage: the case of pumped hydro storage in Germany," Energy Journal-Cleveland, vol. 32, no. 3, p. 59, 2011.

[21] A. Masoumzadeh, E. Nekouei, T. Alpcan, and D. Chattopadhyay, "Impact of Optimal Storage Allocation on Price Volatility in Energy-only Electricity Markets," IEEE Transactions on Power Systems, vol. PP, no. 99, pp. 1-1, 2017.

[22] G. Kirchgässner and J. Wolters, Introduction to modern time series analysis. Springer Science \& Business Media, 2007.

[23] C. S. Park, G. Kim, and S. Choi, Engineering economics. Prentice Hall: Upper Saddle River, NJ, USA, 2007, vol. 22.

[24] B. E. Hobbs, "Linear complementarity models of Nash-Cournot competition in bilateral and POOLCO power markets," IEEE Transactions on Power Systems, vol. 16, no. 2, pp. 194-202, May 2001

[25] W. W. Hogan, "A Market Power Model with Strategic Interaction in Electricity Networks," The Energy Journal, vol. 18, no. 4, pp. 107-141, 1997.

[26] E. G. Kardakos, C. K. Simoglou, and A. G. Bakirtzis, "Optimal bidding strategy in transmission-constrained electricity markets ", Electric Power Systems Research, vol. 109, pp. 141 - 149, 2014.

[27] D. Fudenberg and J. Tirole, Game theory. Cambridge, Mass. : M.I.T. Press, 1991.

[28] W. Gerardi and P. Galanis, "Emissions mitigation policies and security of electricity supply," Jacobs Group (Australia), Tech. Rep., June 2017.

[29] A. Masoumzadeh, E. Nekouei, and T. Alpcan, "Impact of a coal power plant closure on a multi-region wholesale electricity market," in 2017 IEEE PES Innovative Smart Grid Technologies Conference Europe (ISGT-Europe), Sept 2017, pp. 1-6. 


\section{University Library}

\section{- M M I E E R VA A gateway to Melbourne's research publications}

Minerva Access is the Institutional Repository of The University of Melbourne

Author/s:

Masoumzadeh, A;Nekouei, E;Alpcan, T

Title:

Wind Versus Storage Allocation for Price Management in Wholesale Electricity Markets

Date:

2020-04-01

Citation:

Masoumzadeh, A., Nekouei, E. \& Alpcan, T. (2020). Wind Versus Storage Allocation for Price Management in Wholesale Electricity Markets. IEEE Transactions on Sustainable Energy, 11 (2), pp.817-827. https://doi.org/10.1109/TSTE.2019.2907784.

Persistent Link:

http://hdl.handle.net/11343/241557 